\title{
DISPONIBILIDAD Y ACCESO A LOS ALIMENTOS DE POBLADORES RURALES DEL MUNICIPIO DE SAN JOSÉ DE CUSMAPA, MADRIZ EN EL AÑO 2008
}

\author{
Pedro Noel Torrez Rodríguez ${ }^{1}$, Idania del Socorro Montes Blandón ${ }^{2}$ \\ Iris Lucía Manzanares Martínez²
}

${ }^{1}$ Docente - Investigador FDR-UNA, teléfono (505) 2253-1971. E-mail: pedro0104_rodriguez@yahoo.es

${ }^{2}$ Egresadas de la Carrera de Agronegocios, FDR-UNA

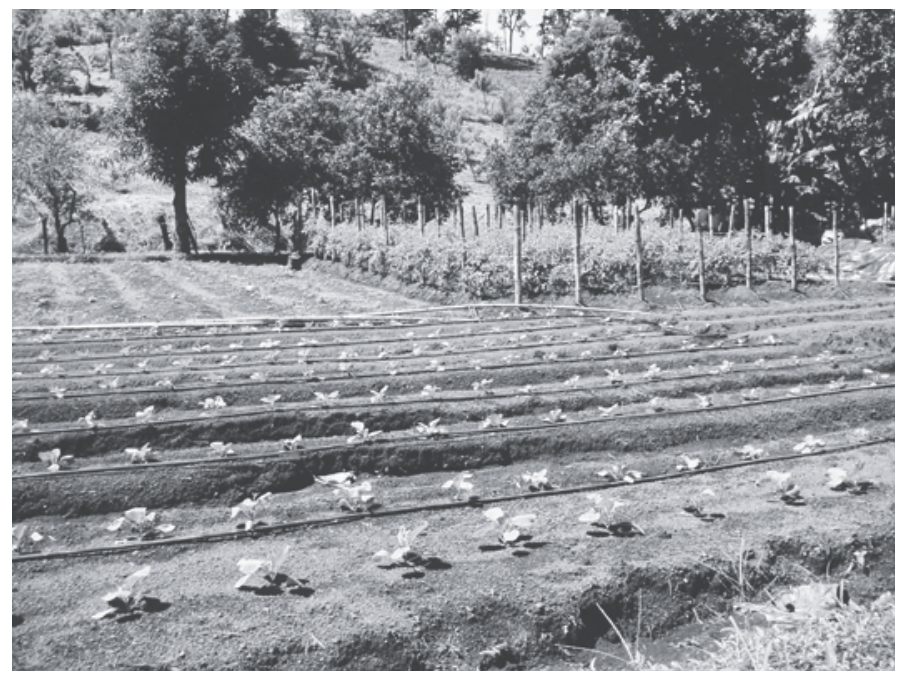

\section{RESUMEN}

El tema disponibilidad y acceso se enmarca dentro de la seguridad alimentaria y nutricional; específicamente, es un análisis de las variables disponibilidad, acceso y aprovechamiento de alimentos de los pobladores rurales del Municipio de San José de Cusmapa (El Mojón, El Rodeo, Los Llanitos, Los Ángeles I, II III, San Francisco de Imires y La Fuente). En el estudio se planteó disponer de información respecto a la situación alimentaria de la localidad, para que los decisores orienten líneas, políticas y estrategias en función de mejorar la calidad de vida de los pobladores rurales del municipio. La metodología utilizada fue participativa con pobladores rurales de San José, líderes comunales, representantes de ONG's, autoridades municipales, del Ministerio de Educación y Salud. Ellos brindaron información que fue recopilada a través de la observación, encuestas y entrevistas que luego, fue analizada y presentada ante las autoridades locales de las cuales surgieron sugerencias que fueron incorporadas al estudio. Se seleccionó a las comunidades de San José de Cusmapa porque representan el $87.1 \%$ de extrema pobreza, según mapa de pobreza de Nicaragua realizado en el 2001. Con relación a la variable disponibilidad, los resultados demostraron que el 71.4\% de la población no logra satisfacer sus necesidades alimenticias, cifra coincidente con el estudio de pobreza del 2001. Respecto al acceso a los alimentos, sólo un $10.2 \%$ de

\section{ABSTRACT}

The topic availability and access was framed within feeding and the Nutritional Security, specifically to the analysis of the variables availability, access and the improvement of food of the rural inhabitants of San José de Cusmapa (El Mojón, El Rodeo and Los Llanitos. Los Angeles I, II, III, San Francisco de Imides and La Fuente), to provide information of the current feeding situation thus to orient strategies to improve the quality of life of the rural inhabitants of the municipal. The methodology used was participatory with rural inhabitants of San Jose, communitarian leaders, NGOs represent in the municipality officials of the Ministry of Education and Health. They supplied information which was collected through observation, surveys, and interviews which were later analyzed and presented to the local officials incorporating their suggestions to this study, rural inhabitants of San Jose de Cusmapa were selects because they represents the $87.1 \%$ of extreme poverty according to the map of poverty of Nicaragua printed in 2001, and about availability the findings showed that $71.4 \%$ did not satisfy their needs, just the $28.6 \%$ satisfies their food needs coinciding with the study carried out in 2001. And concerning access just the $10.2 \%$ has fully and satisfactory access and food, 79. $6 \%$ has a fall access; the $10.2 \%$ meet it insufficiently. And talking about hygiene and improvement of food just 93.9\% make good use of the harvested it their land due to the fact 
pobladores tiene un acceso pleno y suficiente a los mismos; el $79.6 \%$ sólo a medias lo logra, y el $10.2 \%$ lo hace de forma insuficiente. Sobre higiene uso y aprovechamiento de los alimentos, un 93.9\% de la población conoce y hace uso adecuado de los mismos. Ello se explica porque la mayoría de los pobladores han recibido capacitación al respecto. San José de Cusmapa presenta una aguda situación de inseguridad alimentaria, debido a que solo un $28.6 \%$ de sus pobladores dispone de alimentos seguros y estables; un 10.2\% tiene acceso a los alimentos y el 90\% aprovecha y utiliza higiénicamente los alimentos poco disponible y accesible. Los factores de riesgos de la seguridad alimentaria están asociados a las prolongadas sequías, insuficientes factores de producción, bajo rendimiento de cultivos, alta incidencia de plagas y enfermedades, escasez de fuentes de agua y semillas, poca presencia y apoyo de organizaciones y los altos precios de los alimentos.

Palabras clave: Seguridad alimentaria y nutricional, disponibilidad, acceso, higiene y aprovechamiento de alimentos. that most of the inhabitants have received training (61.2\% of the people surveyed) on hygiene, use, and manipulation of food. San José de Cusmapa presents an acute situation of food insecurity, due that just $28.6 \%$ of the inhabitants of the zone has at their at disposal secure and stable food, $10.2 \%$ has access to food and $90 \%$ make hygienically good use of the few that has at their disposal and access. The risk factors of food security are closely related to the prolonged droughts, inadequate factors of production, low yields, high incident of plagues and diseases, short of water supplier and seeds, little presence and support of organizations and high price of foods.

Keywords: Feeding and nutritional security, availability, access, hygiene and make good use of food.

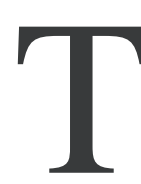
ener seguridad alimentaria significa poder obtener en todo momento los alimentos requeridos para llevar una vida activa y sana. La Seguridad Alimentaria y Nutricional (SAN) dependen de tres pilares o puntos de sustentación. Debe haber alimentos suficientes, disponibles y accesibles para todos y saber utilizar de la mejor manera posible los alimentos disponibles (FAO 2003).

Los países de Centroamérica presentan condiciones heterogéneas en sus indicadores de SAN. Los actuales índices de desarrollo humano, de subalimentación y de desnutrición evidencian que, a pesar de los avances en materia de SAN, Centroamérica sigue siendo una región con grandes grupos de población afectados por la subalimentación y la desnutrición crónica y aguda. En Guatemala, El Salvador, Honduras y Nicaragua, grandes grupos de personas sobreviven en condiciones de extrema pobreza y hambre; es decir, sin recursos suficientes para asegurar diariamente sus necesidades de alimentación y nutrición (INCAP CESNA, PRESANCA, 2008).

La disponibilidad de alimentos, se refiere a qué tipo de alimentos puede encontrar un ser humano a escala local, regional o nacional. Esta disponibilidad se determinada por la producción de alimentos que se da tanto en el ámbito local como nacional, así como de aquellos que procedan del extranjero (INCAP, 2006).

La FAO se refiere a acceso y control alimentario cuando existe una relación estrecha del hombre con los medios de producción (tierra, agua, insumos, tecnología, conocimiento, etc.). En este sentido, los alimentos deben ser accesibles para todos y todas, ser distribuidos y estar disponibles localmente.

La utilización biológica está relacionada con el estado nutricional, como resultado del uso individual de los alimentos (ingestión, absorción y utilización). La inadecuada utilización biológica puede tener como consecuencia la desnutrición o la mal nutrición. Con frecuencia, se toma como referencia el estado nutricional de los niños y las niñas, pues las carencias de alimentación o salud en estas edades, tienen graves consecuencias a largo plazo y a veces permanentes (FAO, 2006).

El 18 de junio del 2009 se aprobó la ley de seguridad y soberanía alimentaria cuyo objetivo es garantizar el derecho de los nicaragüenses a tener alimentos suficientes, inocuos y nutritivos acorde a sus necesidades vitales. Asimismo en la ley se establece que el Estado promoverá programas que aseguren una adecuada disponibilidad de alimentos y una distribución equitativa de los mismos; entre otras cosas establece recursos provenientes del presupuesto general de la república que aseguren la producción alimentaria en el país.

En Nicaragua uno de los principales problemas que enfrentan los pobladores de las zonas rurales es la desnutrición alimentaria debido al acceso y consumo inadecuado de alimentos. El Municipio de San José de Cusmapa se ubica en el Departamento de Madriz, siendo uno de sus problemas principales la disponibilidad, aprovechamiento y acceso de los productos alimenticios (Mendoza, 2008).

Los problemas que afrontan las comunidades del Municipio de San José de Cusmapa se pueden agrupar en 
relación con la satisfacción de necesidades básicas, tales como: alimentación, agua potable, energía eléctrica, vías de acceso, entre otros; y en un segundo grupo aquellos que tienen una relevancia estratégica como: escasez de tierra para cultivar, falta de financiamiento para emprender proyectos productivos, falta de fuentes de empleo e ingresos, deforestación y recuperación de fuentes de agua, así como la falta de valor agregado de productos, sin perder de vista los efectos negativos ocasionados por factores del clima como las frecuentes sequías (Zapata 2008).

San José de Cusmapa es uno de los municipios que posee la tasa más alta de desnutrición a escala nacional debido a la falta de capacitación y conocimiento sobre Seguridad Alimentaria y Nutricional; también por la poca asistencia de las ONG'S a este municipio, explicable en parte porque éste se encuentra un poco aislado (Zapata, 2008).

Para hacer el estudio, se seleccionó a los pobladores rurales de San José de Cusmapa porque soportan un 87.1\% de extrema pobreza, ello según estudio del mapa de pobreza de Nicaragua realizado en el 2001.

Las principales actividades productivas que se realizan en el municipio son: producción de granos básicos (maíz, fríjol, millón) para autoconsumo; hortalizas (riego), parches de café, ganadería, transformación de la madera (Pino) muy poco y fabricación de artesanías (Zapata, 2008).

El presente trabajo es un análisis de disponibilidad y aprovechamiento de alimentos de los pobladores rurales de las comunidades El Rodeo, Los Llanitos, Los ángeles I, II y III, San Francisco de Imires y La Fuente del municipio de San José de Cusmapa, del Departamento de Madriz. El estudio permite hacer una valoración del nivel de suficiencia alimentaria, información que permitirá a los tomadores de decisiones reorientar estrategias, recursos y acciones para la seguridad y soberanía alimentaria en función de mejorar la calidad de vida de los pobladores locales.

\section{MATERIALES Y MÉTODOS}

La investigación realizada es de tipo descriptivo, haciendo uso de elementos de la metodología cualitativa y cuantitativas con datos primarios y fuentes secundarias, habiéndose seleccionado ocho (8) comunidades (El Mojón, El Rodeo, Los Llanitos, Los Ángeles I, II, III, San Francisco de Imires y La Fuente) de un total de 26; selección que obedece a cercanía, acceso y número de pobladores en una muestra de 49 usuarios de 7532 habitantes con un margen de error del $2 \%$.
El análisis estuvo enfocado en los factores que inciden en la Seguridad Alimentaria y Nutricional, basado en las variables disponibilidad, acceso y aprovechamiento para determinar el grado de autonomía o seguridad alimentaria en los pobladores rurales de San José de Cusmapa.

El Municipio de San José de Cusmapa, que en lengua chorotega significa BALCÓN DE ALTAS MONTAÑAS, se localiza geográficamente entre las coordenadas $13^{\circ} 17^{\prime \prime}$ de latitud norte y a $86^{\circ} 39^{\prime}$ de longitud oeste. Está inserta en el Departamento de Madriz, comprende 26 comunidades y tres sectores urbanos, los cuales, para efectos de administración del municipio, se subdividen en 8 micros regiones ( $\mathrm{La}$ Jabonera, El Carrizo, El Apante, San Francisco de Imires, El Naranjo, El Terrero, Los Llanitos y Cusmapa urbano). La Cabecera Departamental Somoto se localiza a 34 kilómetros al suroeste del Departamento de Madriz. El casco urbano de San José de Cusmapa se encuentra a una elevación de 1280 metros sobre el nivel del mar, siendo esta una particularidad por ser la cabecera municipal con mayor elevación de la República de Nicaragua y con una extensión territorial de $98 \mathrm{~km}^{2}$. Limita al Norte con el Municipio de Las Sabanas; al Sur, con los Municipio de San Juan de Limay (Estelí) y San Francisco del Norte (Chinandega.); al Este, con el municipio de San Juan de Limay (Estelí); al Oeste con el Municipio San Francisco del Norte (Chinandega) y la República de Honduras.

Pasos metodológicos. Delimitación del tema con participación de representantes de la Universidad Politécnica de Madrid (UPM) y de la Facultad de Desarrollo Rural de la Universidad Nacional Agraria (UNA).

Revisión bibliográfica que comprende libros, revistas, guías, páginas de Internet.

Establecimiento de contactos con líderes comunitarios y pobladores del Municipio de San José de Cusmapa, con el objetivo de recopilar información primaria y así definir las comunidades del estudio (representantes de la Alcaldía Municipal, el Programa Regional de Seguridad Alimentaria y Nutricional para Centroamérica (PRESANCA). Este paso facilitó la validación de instrumentos de información como entrevistas y encuestas.

Trabajo de campo a través de entrevistas dirigidas a líderes, pobladores y representantes de organismos establecidos en la localidad, así como encuestas a pobladores de la misma zona.

Procesamiento de la información a través del programa estadístico SPSS y tablas de Excel. 
Incorporación de sugerencias y reproducción de los documentos finales.

\section{RESULTADOS}

Disponibilidad. El estudio refleja que, en las comunidades de San José de Cusmapa, existe una insuficiente e inestable disponibilidad de alimentos (el $71.4 \%$ no satisface sus necesidades alimentarias), esto es debido a condiciones climáticas adversas como las prolongadas sequías (7 meses), escasez de agua, presión sobre los recursos naturales, alta incidencia de plagas en los cultivos, déficit de factores de producción como tierras (1.30 mz promedio por familia), 60\% de tierras sin títulos de probiedad. escasa diversificación (friiol. granos básicos con alto contenido de carbohidratos pero con deficiencia en proteínas, vitaminas y minerales.

Acceso. Por acceso la FAO define la relación que los pobladores guardan con los medios de producción (tierra, agua, insumos, tecnología, conocimiento, etc.), en función de obtener alimentos que deben ser accesibles, para lo cual se requiere que dichos recursos estén disponibles localmente para todos y todas.

Con ingresos propios muy exiguos (C\$ 2506.25 como promedio mensual), las comunidades de San José de Cusmapa apenas logran acceder a una tercera parte de la canasta básica, valorada en C\$ 8424 al mes de diciembre del 2008. En los dos últimos años (2007-2008), los brecios de la canasta básica se incrementaron entre el

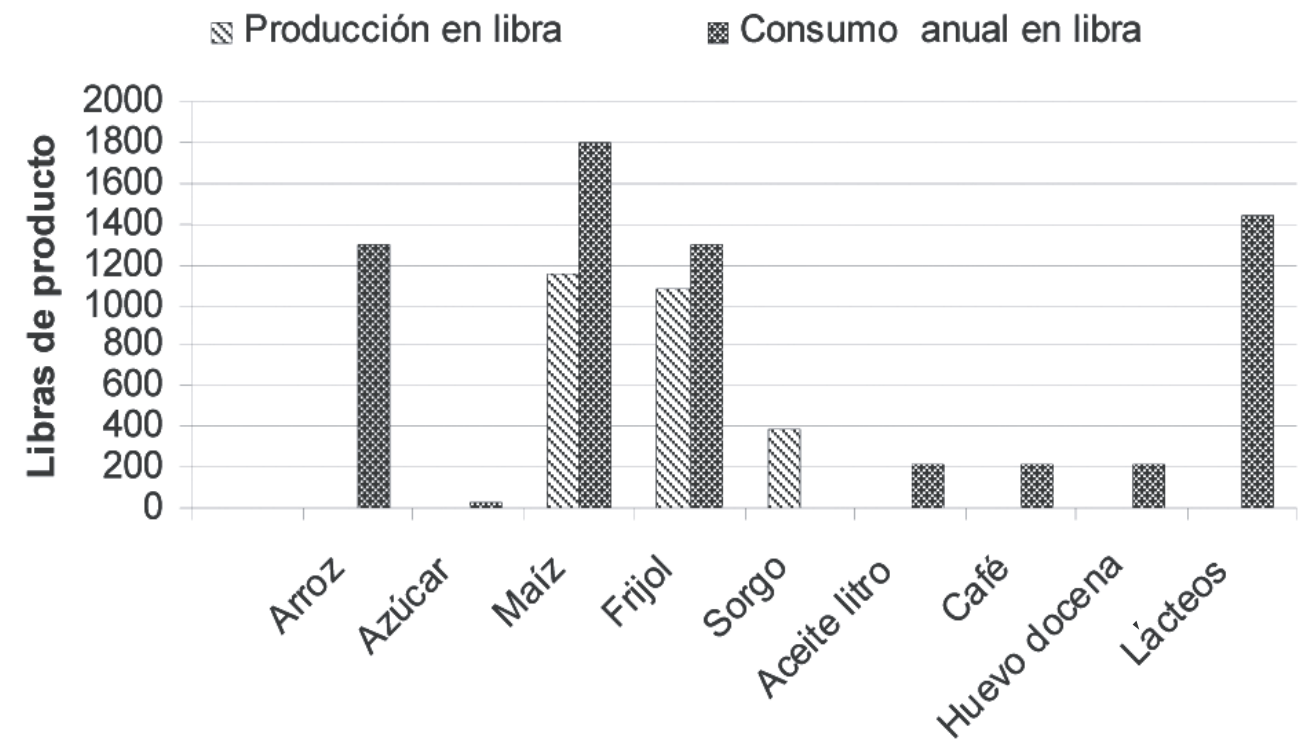

Figura 1. Balance producción disponible-consumo anual de alimentos.

maíz, maicillo), bajos rendimientos, escasez de fuentes alternativas de empleo, no acceso al crédito y carencia de centros de abastecimiento y comercialización en la localidad.

Sólo un 40\% de la población tiene acceso al crédito (utilizado en función de la producción de granos básicos), gracias a que tiene legalizada su propiedad que, como promedio por familia, posee una extensión de 1.30 manzanas. El 60\% restante, no tiene acceso a este crédito dado que los rubros a los que se dedica son de alto riesgo. Por la misma razón, tampoco recibe apoyo de otro tipo de instituciones.

Debido a las condiciones climáticas, junio y agosto son los meses de mayor déficit alimentario. Ángeles II e Imires presentan una situación crítica en la disponibilidad de alimentos, que se agudiza justamente entre esos meses. El alimento básico de estas familias son los
$156 \%$ y el $180 \%$. Por si fuera poco, las alternativas de empleo local son casi nulas, no existen centros de abasto y distribución de alimentos.

El costo incremental de la canasta básica nacional del 2006 al 2008, fue respectivamente de C\$2 938.00, 7539.00 y 8242.00 . Los ingresos promedios mensuales al 2008, provenientes de la actividad agrícola, fueron de C\$ 2 506.25; lo que permitió acceder a tan solo el 30.4\% de la canasta básica nacional.

Con ingresos promedios mensuales de C\$ 2506.25 y un costo promedio de la canasta básica local de 9 productos (equivalentes a C $\$ 1667.01$ a diciembre del 2008); se adquiere 1.5 canastas básicas, sin incluir cárnicos, vestuario y gastos del hogar; limitándose así sólo a maíz, frijol, arroz, azúcar, café, aceite, sal, huevos y lácteos. 


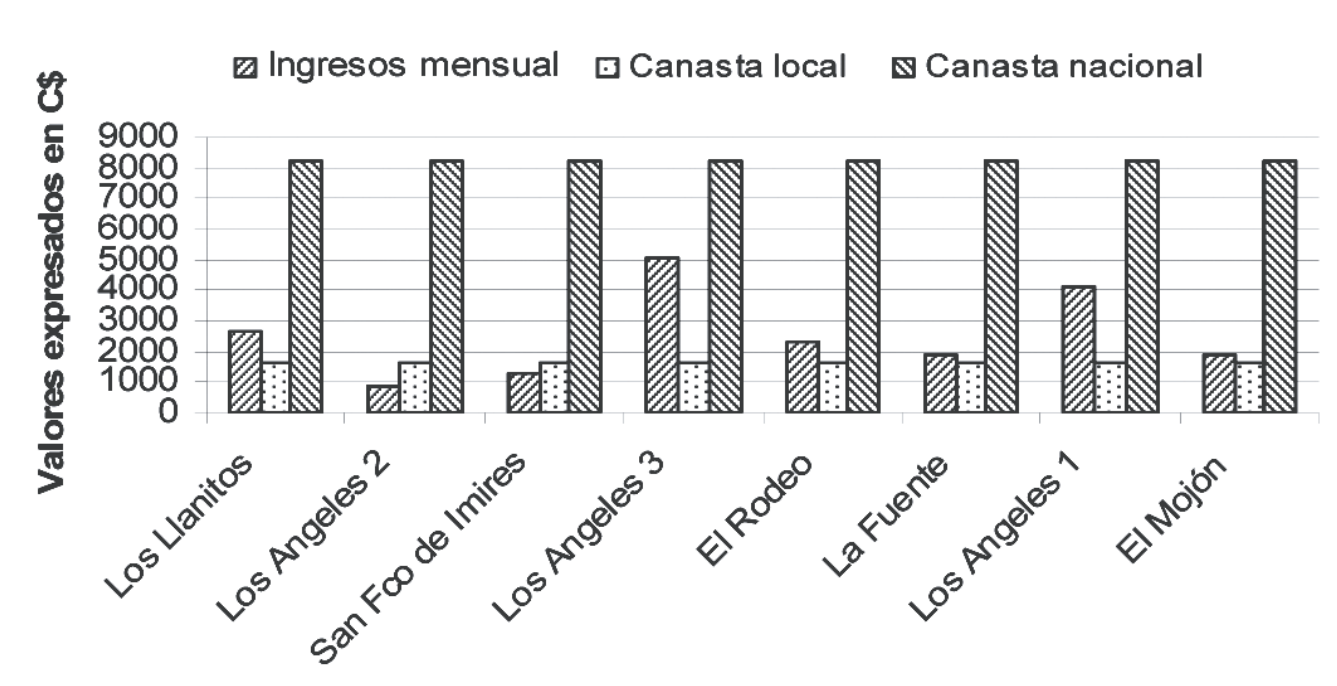

Figura 2. Acceso a la canasta básica diciembre 2008.

Tan sólo el 10.2\% de los pobladores de la zona tiene acceso a sus alimentos; $79.6 \%$ accede de forma medianamente suficiente y el $10.2 \%$ restante de modo insuficiente, fenómeno que se explica por la escasez de agua y la irregularidad de las lluvias; por el pobre acceso al crédito, los bajos rendimientos de producción, el alto precio de los alimentos, la escasez de semilla, la falta de apoyo por parte de las organizaciones que tienen presencia en la zona, la alta incidencia de plagas en los cultivos y, sobre todo, porque un 60\% de la población carece de tierras propias.

Los Ángeles II y San Fco de Imires son las comunidades que presentan un acceso crítico a la canasta básica local.
En temporadas bajas, para poder subsistir, los pobladores venden su fuerza de trabajo en las plantaciones de café, emigran temporalmente a países vecinos; la mujer cuando encuentra oportunidades, labora como doméstica o vende cuajadas y hornado a base de maíz

Aprovechamiento y consumo. El INCAP y la OPS anotan que el consumo y aprovechamiento de los alimentos dependen de las costumbres, las prácticas de alimentación, el estado de salud de las personas y de la forma de almacenar, preparar, aprovechar y distribuir los alimentos en la familia.

Los resultados a que hemos llegado con el presente estudio reflejan que un 93.9\% manipula y aprovecha adecuadamente los alimentos producidos en sus parcelas, en cambio el 6.1\% no aplica medidas de ningún tipo ni

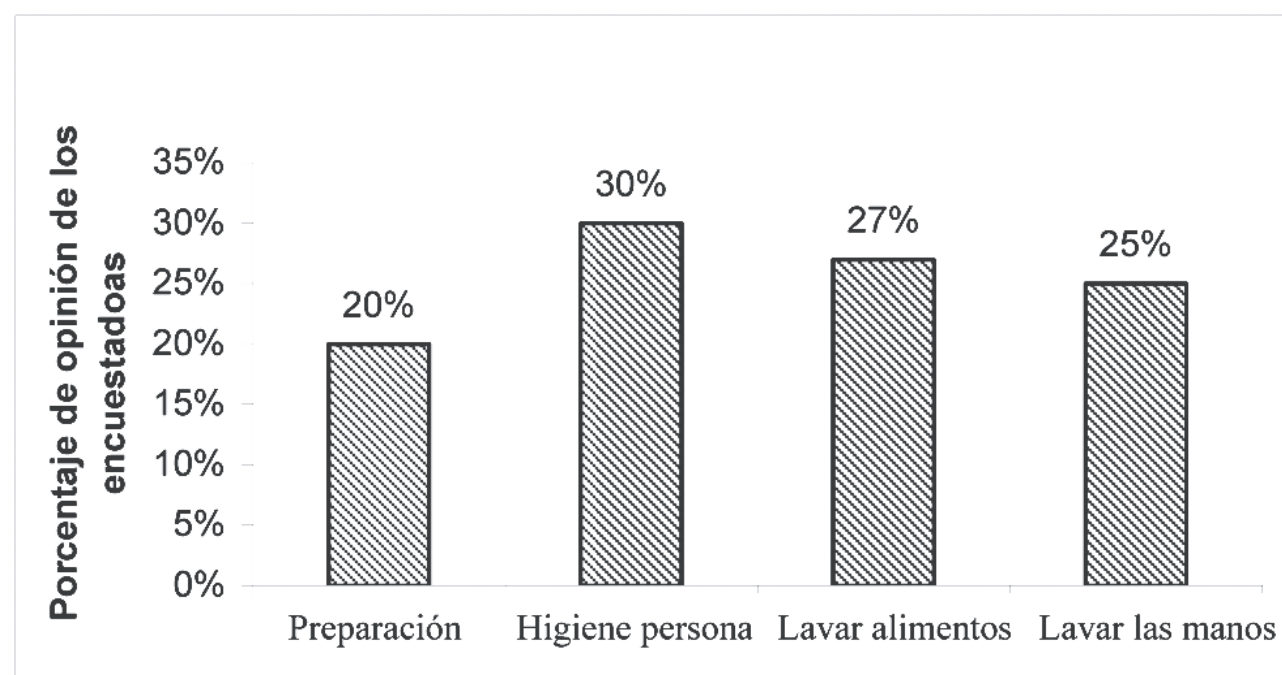

Figura 3. Factores que influyen en el aprovechamiento e higiene de los alimentos. 
hace el uso necesario de los mismos; sin obviar que existe déficit alimentario y desnutrición crónica en la zona.

Como resultado de las capacitaciones recibidas, un $71.4 \%$ de los habitantes conocen los factores que influyen en la higiene y aprovechamiento de los alimentos; posee conocimiento sobre seguridad alimentaria y nutricional (SAN) y saben manejar y manipular los alimentos.

La alimentación está basada en carbohidratos y esporádicamente se agregan proteínas cuando se consume huevo, leche y cuajada.

\section{CONCLUSIONES}

San José de Cusmapa presenta una aguda situación de inseguridad alimentaria, debido a que solo un $28.6 \%$ de pobladores de la zona dispone de alimentos seguros y estables, un $10.2 \%$ tiene acceso a los alimentos y el $90 \%$ aprovecha y utiliza higiénicamente lo poco que dispone y accede.

Los factores de riesgos de la seguridad alimentaria están asociados a las prolongadas sequías, insuficientes factores de producción, bajos rendimiento de los cultivos, alta incidencia de plagas y enfermedades, escasez de fuentes de agua y semillas, poca presencia y apoyo de organizaciones y altos precios de los alimentos.

Con relación a la variable disponibilidad, los resultados demostraron que el $71.4 \%$ no logra satisfacer sus necesidades, solo un $28.6 \%$ satisface sus necesidades alimenticias debido a factores económicos, técnicos, climáticos, tierras marginales, escases de semillas, presencia de plagas y altos precios de los alimentos.

Los recursos disponibles no son suficientes para garantizar una disponibilidad de alimentos de forma suficiente y permanente en cantidad y calidad, tampoco existen fuentes alternativas de empleo local; debiendo buscar otras alternativas de ingreso como la venta de fuerza de trabajo en temporada de verano y emigración a países vecinos.

Respecto al acceso solo un $10.2 \%$ de pobladores tiene acceso pleno y suficiente a los alimentos, el 79.6\% accede a los mismos a medias y el 10.2\% lo hace de forma insuficiente por razones de bajos ingresos, alto costo de la canasta básica y escasez de fuentes alternativas de empleo.

De ocho comunidades estudiadas, Ángeles II e Imires se encuentran en situación crítica respecto al acceso a la alimentación con incidencia directas en el nivel de vida de los pobladores.

Sobre la higiene y aprovechamiento un 93.9\% aprovecha adecuadamente los alimentos producidos en sus parcelas, debido a que la mayoría de los pobladores han recibido capacitación (61.2\% de los encuestados) sobre higiene, uso y manipulación de alimentos.

\section{RECOMENDACIONES}

Promover e impulsar la producción de alimentos mediante huertos caseros, incorporando nuevos rubros para mejorar la disponibilidad en cantidad y calidad de alimentos, en correspondencia a la ley de soberanía y seguridad alimentaria nacional.

Establecer vínculos y enlaces entre entidades gubernamentales y pobladores del sector rural para que legalicen la situación de sus propiedades y ser sujetos de crédito, acceder a mejores oportunidades de producción e ingresos económicos.

Priorizar a las comunidades Ángeles II y San Fco de Imires en los programas de apoyo técnico social y productivo por ser las comunidades de mayor nivel de pobreza.

Mejorar las vías de acceso a las comunidades rurales, para que los productores puedan sacar sus cosechas y comercializar sus productos.

Identificar iniciativas, formular y gestionar planes y programas de agronegocios para la autogeneración de ingresos y empleo productivo.

Promover en los sistemas de producción métodos y técnicas de recolección y almacenamiento de agua, producción y almacenamiento de semillas mejoradas.

Establecimiento de centros de acopio de granos básicos.

Promoción del agroturismo y la agroindustria de producción local.

Priorizar la legalización de las tierras de los micros y pequeños productores

Establecer redes y alianzas entre los pobladores de la zona, para que puedan acceder a los programas de gobierno, ONG's e instituciones que apoyan a grupos vulnerables.

Impulsar el agroturismo, para dar a conocer la cultura del municipio a nivel nacional, mejorar su nivel de vida y reducir la migración.

Priorizar la seguridad alimentaria y nutricional en mujeres embarazadas y niños (as) menores de dos años, como un buen punto de partida para la buena nutrición, el bienestar y la calidad de vida de los grupos en desventaja.

Promoción de proyectos de construcción de caminos y viviendas con mano de obra local.

Establecer redes de producción y comercialización de productos agrarios.

Promover proyectos comunitarios de producción alimentaria. 
Fortalecer las redes y alianzas con organismos e instituciones que se dedican a promover la educación, salud, vivienda, seguridad alimentaria, organización, producción y medio ambiente

Capacitación para la diversificación alimentaria y productiva.

\section{AGRADECIMIENTOS}

A la Universidad Politécnica de Madrid, España, por financiar proyectos de investigación que realizan docentes y estudiantes de la Facultad de Desarrollo Rural de la Universidad Nacional Agraria, así como a los pobladores de las comunidades de San José de Cusmapa por su desinteresado apoyo para con los estudiantes de nuestra facultad.

\section{REFERENCIAS BIBLIOGRÁFICAS}

Banco Mundial; ASDI (Agencia Sueca para el Desarrollo Internacional), NORAD (Agencia Noruega de Cooperación al Desarrollo), PNUD (Programa de las Naciones Unidas para el Desarrollo). 2004. Análisis de pobreza y seguridad alimentaria y nutricional en Nicaragua.

Castilblanco, J. 2004. Cuestionario para el registro de productores FLO-INTERNACIONAL. CRS (Catholic Relief Services), Managua, NI.

CIPRES (Centro para la Promoción, la Investigación y el Desarrollo Rural y Social, NI). 2001. Programa productivo alimentario para combatir el hambre y la pobreza. Managua, Nicaragua.

FAO (Organización de las Naciones Unidas para la Agricultura y la Alimentación). 2002. Cadena de seguridad alimentaria y nutricional.

FAO-PESA. (Organización de las Naciones Unidas para la Agricultura y la Alimentación), (Programa Especial para la Seguridad Alimentaria). 2008. Programa especial de seguridad alimentaria y nutricional y la organización para la alimentación y la agricultura.

Hernández Sampieri, R. 2002. Metodología de la investigación México: McGraw Hill.

INCAP. 2006. Instituto de nutrición en Centroamérica y Panamá, 25 p.

INCAP, OPS. 2005. Generalidades de seguridad alimentaria y nutricional.

Mendoza, E. 2007. Monografía sociocultural Municipio San José de Cusmapa, Madriz.

PRESANCA 2008. Programa regional de Seguridad Alimentaria y Nutricional para Centroamérica.

Zapata, LE. 2008. Entrevista Alcaldía de San José de Cusmapa, Somoto, Nicaragua. 